\title{
Some Properties of the Attainable Set for the Abstract Control Problem with Application to Controllability
}

\author{
B. Shklyar
}

Abstract. For linear differential control system described by $\dot{x}=A x(t)+B u(t), x(0)=$ $x_{0}, y(t)=C x(t)$ ( $A$ generates a strongly continuous semigroup $\{S(t)\}_{t \geq 0}$ on a Banach space $X ; B$ and $C$ are bounded operators) the attainable set

$$
K(t)=\left\{\dot{x} \in X: x=\int_{0}^{t} S(t-\tau) B u(\tau) d \tau \text { for some } u(\cdot) \in L_{2}([0, t], U)\right\}
$$

and the unobservable set

$$
N(t)=\{x \in X: C S(\tau) x \equiv 0 \text { for all } \tau \in[0, t]\}
$$

are investigated. Conditions of the independence of $t$ for closure $\overline{K(t)}$ and $N(t)$ have been established.

Keywords: Attainable sets, controllability, abstract evolution equations, linear hereditary systems

AMS subject classiflcation: Primary $35 \mathrm{R} \mathrm{30,} \mathrm{secondary} 35 \mathrm{~J} 20,65 \mathrm{M} 30$

\section{Introduction}

We consider a system described by linear abstract differential equation of evolution type

$$
\begin{aligned}
\dot{x}(t) & =A x(t)+B u(t) \\
x(0) & =x_{0} \\
y(t) & =C x(t) \quad\left(0 \leq t \leq t_{1}\right)
\end{aligned}
$$

where $X, Y, U$ are Banach spaces, $x(t) \in X$ is the current state, $x_{0} \in X$ is the initial state, $y(t) \in Y$ is the output, $u(t) \in U, u(\cdot) \in L_{2}\left(\left[0, t_{1}\right], U\right)$ is the control, $A$.is a linear operator generating a strongly continuous semigroup $\{S(t)\}_{t \geq 0}$ of operators in the class $C_{0} ;$ and $B: U \rightarrow X$ as well as $C: X \rightarrow Y$ are linear bounded operators.

B. Shklyar: Bar-Ilan Univ., Dept. Math., Ramat Gan 52900, Israel 
The weak solution $x(t)$ of equation (1)-(2) is evaluated by the formula (see [1: Theorem 4.8.3])

$$
x(t)=S(t) x_{0}+\int_{-0}^{t} S(t-\tau) B u(\tau) d \tau .
$$

The attainable set $K(t)$ for equation (1)-(2) is defined as

$$
K(t)=\left\{x \in X: x=\int_{0}^{t} S(t-\tau) B u(\tau) d \tau \text { for some } u(\cdot) \in L_{2}([0, t], U)\right\}
$$

The unobservable set $N(t)$ for équation (1)-(2) is defined as

$$
N(t)=\{x \in X: C S(\tau) x \equiv 0 \text { for all } \tau \in[0, t]\}
$$

Attainable sets, unobservable sets and their propertics play an important role in the controllability, observability, stabilizability and optimal control theory for linear controllable systems. Properties of these sets and formulas for representation of solutions of controllable systems are one of the main research tools in various fields of linear control theory. Properties of attainable sets have been investigated in a number of papers devoted to controllability, observability and optimal control problems for delay systems (see, for instance, $[2-4,7,10,12,15,17,20]$, which can be considered as a particular case of the abstract linear evolution control system [9].

The approximate null-controllability criterion has been obtained in [21] for abstract control systems with both distributed and boundary control (the abstract boundary control problem). Among restrictions for the considered control problems, the following one holds:

The attainable set of the system under consideration does not depend on time at least for sufficiently large values of time.

The object of the present article is to establish the independence of $t$ for the attainable set $K(t)$ and (by duality) for the unobservable set $N(t)$ for a wide class of abstract linear control problems with bounded input-output operators, provided $t$ is sufficiently large.

\section{Basic assumptions}

If $x \in X$ and $f \in X^{*}$, we will write $(x, f)$ instead of $f(x)$. The upper superscript ${ }^{\top}$ denotes transposition.

As usual $\mathbb{R}$ is the set of real and $\boldsymbol{C}$ the set of complex numbers.

For any set $K \subset X$ we denote by $\bar{K}$ the closure of $K$ with respect to the uniform topology of $X$ and by $K^{\perp}$ the set

$$
K^{\perp}=\left\{y \in X^{*}:(x, y)=0 \text { for all } x \in K^{*}\right\} .
$$


We assume the operator $A$ to have the following properties (I) - (IV):

(I) The domain $D\left(A^{*}\right)$ is dense in $X^{*}$.

(II) The operator $A$ has a purely point spectrum $\sigma$ which is either finite or has no finite limit points and each $\lambda \in \sigma$ is of a finite multiplicity.

Let the numbers $\lambda_{j} \in \sigma(j \in \mathbb{N})$ be enumerated in the order of non-decreasing absolute values, let $\alpha_{j}$ be the multiplicity of $\lambda_{j} \in \sigma$, and let

$$
\begin{gathered}
\varphi_{j k l} \quad \text { and } \quad \psi_{j k l} \\
\left(j \in \mathbb{N} ; k=1, \ldots, m_{j} ; l=1,2, \ldots, \beta_{j k} ; \sum_{k=1}^{m_{j}} \beta_{j k}=\alpha_{j}\right)
\end{gathered}
$$

be the generalized eigenvectors of the operators $A$ and $A^{*}$, respectively, such that

$$
\begin{gathered}
\left(\varphi_{j p \beta_{p}-l+1}, \psi_{k s q}\right)=\delta_{j k} \delta_{p s} \delta_{l q} \\
\left(j, k \in \mathbb{N} ; p=1, \ldots, m_{j} ; l=1, \ldots, \beta_{j p} ; s=1, \ldots, m_{k} ; q=1, \ldots, \beta_{k s}\right) .
\end{gathered}
$$

(III) There exists a time moment $T \geq 0$ such that for all $x \in X$ and $t>T$ the function $x(t)=S(t) x$ is expanded in a series of generalized eigenvectors of the operator $A$, converging for a certain grouping of terms uniformly with respect to $t$ on an arbitrary interval $\left[T_{1}, T_{2}\right] \quad\left(T_{1}>T\right)$.

(IV) A sequence $\left\{x_{i}\right\}_{i \in \mathbb{N}}$ of functions from $L_{2}^{\text {loc }}[0,+\infty)$ is called minimal on $[0, \nu](\nu>0)$ if there is a sequence $\left\{y_{j}\right\}_{j \in \mathbb{N}}$ of functions from $L_{2}[0, \nu]$ such that

$$
\int_{0}^{\nu}\left(x_{i}(t) y_{j}(t)\right) d t=\delta_{i j} \quad(i, j \in \mathbb{N})
$$

where $\delta_{i j}$ is the Kronecker symbol. The sequence $\left\{y_{j}\right\}_{j \in \mathbb{N}}$ is called a sequence biorthogonal to the sequence $\left\{x_{j}\right\}_{j \in \mathbb{N}}$.

Assume the sequence $\left\{f_{j k}\right\}_{j k}$ of functions

$$
f_{j k}(t)=(-t)^{k} \exp (-\lambda, t) \quad\left(j \in \mathbb{N} ; k=1, \ldots, \alpha_{j} ; t \in[0,+\infty)\right)
$$

to be minimal on $[0, \nu]$. 


\section{Main results}

Our main task consists of establishing conditions for independence of $K(t)$ at least for sufficiently large $t$. The following theorem shows the truth of this property provided assumptions (I) - (IV) take place. Recall that the number $\nu$ was introduced in property (IV).

Theorem 1. If $t_{1} \leq t_{2}$, then $K\left(t_{1}\right) \subseteq K\left(t_{2}\right)$. Further, $\overline{K\left(t_{1}\right)}=\overline{K\left(t_{2}\right)}$ if $t_{1}, t_{2}>$ $T+\nu$.

Proof. Let $t_{1}<t_{2}$ and $x \in K\left(t_{1}\right)$. In this case there exists a control $u_{1}(\cdot) \in$ $L_{2}\left(\left[0, t_{1}\right], U\right)$ such that

$$
x=\int_{0}^{t_{1}} S\left(t_{1}-\tau\right) B u_{1}(\tau) d \dot{\tau} .
$$

Let

$$
\begin{array}{ll}
u_{2}(\tau)=0 & \left(0 \leq \tau<t_{2}-t_{1}\right) \\
u_{2}(\tau)=u_{1}\left(t_{1}-t_{2}+\tau\right) & \left(t_{2}-t_{1} \leq \tau \leq t_{2}\right) .
\end{array}
$$

By'simply computations we obtain

$$
x=\int_{0}^{t_{2}} S\left(t_{2}-\tau\right) B u_{2}(\tau) d \tau
$$

and $u_{2}(\cdot) \in L_{2}\left(\left[0, t_{2}\right], U\right)$. Hence, $K\left(t_{1}\right) \subseteq K\left(t_{2}\right)$ for all $t_{1}<t_{2}$.

Now we will prove inclusion $K\left(t_{2}\right) \subseteq K\left(t_{1}\right)$ for all $T^{\prime} \leq t_{1}<t_{2}$. Let $g \in K\left(t_{1}\right)^{\perp}$ and $R(\mu)=(\mu I-A)^{-1}$ for $\mu \notin \sigma$. By [7: Proposition 2.1]

$$
(S(t) B u, g) \equiv 0^{*} \quad \text { for all } t \in\left[0, t_{1}\right] .
$$

Let $c_{j}$ be a closed contour in the complex plane containing $\lambda_{j}$ but not $\lambda_{k}$ for $k \neq j$. Consider the linear bounded operator

$$
P_{j} x=\frac{1}{2 \pi i} \oint_{c_{j}} R(\xi) x d \xi .
$$

Operator $P_{j}$ is a projector (see [11: Chap. VII, §9). The subspace $X_{j}=P_{j} X$ is called the generalized eigenspace of $A$ at $\lambda_{j} \in \sigma(j \in \mathbb{N})$. Let

$$
\begin{aligned}
\Phi_{j} & =\left\{\varphi_{j k 1}, \varphi_{j k 2}, \ldots, \varphi_{j k \beta_{j k}}\right\} \\
\Psi_{j} & =\left\{\psi_{j k 1}, \psi_{j k 2}, \ldots, \psi_{j k \beta_{j k}}\right\} \\
\left(\Phi_{j}, g\right) & =\left\{\left(\varphi_{j k 1}, g\right), \ldots,\left(\varphi_{j k \beta_{j k}}, g\right)\right\} \\
\left(x, \Psi_{j}\right) & =\left\{\left(x, \psi_{j k 1}\right), \ldots,\left(x, \psi_{j k \beta_{j k}}\right)\right\}
\end{aligned} \quad\left(k=1, \ldots, m_{j}\right)
$$

Choosing the generalized eigenvectors

$$
\varphi_{j k 1}, \varphi_{j k 2}, \ldots, \varphi_{j k \beta_{j k}} \quad\left(k=1, \ldots, m_{j}\right)
$$


of $A$ at $\lambda_{j}$ as a basis in $X_{j}$, we obtain

$$
S(t) P_{j} x=\Phi_{i} \exp \left(\Lambda_{j} t\right) x_{j}
$$

where

$$
\Lambda_{j}=\left\{\begin{array}{cccc}
\lambda_{j} & 1 & \ldots & 0 \\
0 & \lambda_{j} & \ldots & 0 \\
\vdots & \vdots & . & \vdots \\
0 & 0 & \ldots & 1
\end{array}\right\}
$$

is the Jordan $\left(\alpha_{j} \times \alpha_{j}\right)$-matrix and

$$
x_{j}=\left(x, \Psi_{j}\right)^{\top} \quad(j \in \mathbb{N}) .
$$

By elementary computations we can obtain

$$
\left.\left(S(t) P_{j} B u, g\right) \equiv\left(\Phi_{j}, g\right) \exp (\Lambda, t)\left(B u, \Psi_{j}\right)^{\top} \quad \text { for all } t \in[0, \infty) \text { and } u \in U\right)
$$

hence, it follows from property (III), (4) and (10) that

$$
\sum_{j=1}^{\infty}\left(\Phi_{j}, g\right) \exp (\Lambda, t)\left(B u, \Psi_{j}\right)^{\top} \equiv 0 \quad \text { for all } t \in\left(T, t_{1}\right] \text { and } u \in U
$$

Obviously, for each $j \in \mathbb{N}$, the function

$$
\left(\Phi_{j}, g\right) \exp \left(\Lambda_{j}\left(t_{1}-t\right)\right)\left(B u, \Psi_{j}\right)^{\top} \quad\left(0 \leq t \leq t_{1}\right)
$$

is a linear combination of functions (6). If $t_{1}>T+\nu$, then, multiplying both parts of (11) by the corresponding elements of the biorthogonal system for (6) and integrating from 0 to $\nu$, we obtain all the coefficients of these linear combinations equal zero. Therefore,

$$
\left(\Phi_{j}, g\right) \exp (\Lambda, t)\left(B u, \Psi_{j}\right)^{\top} \equiv 0 \quad \text { for all } 0 \leq t<\infty \text { and } u \in U
$$

Using this fact and property (III), we obtain $(S(t) B u, g) \equiv 0$ for all $t>T+\nu$ and $u \in U$. The latter identity and (7) imply the inclusion $g \in K\left(t_{2}\right)^{\perp}$. Thus, $K\left(t_{1}\right)^{\perp} \subseteq$ $K\left(t_{2}\right)^{\perp}$. Hence $\overline{K\left(t_{2}\right)} \subseteq \overline{K\left(t_{1}\right)}$. Since $K\left(t_{1}\right) \subseteq K\left(t_{2}\right)$ for all $t_{1}$ with $t_{1}<t_{2}$, we obtain $\overline{K\left(t_{1}\right)}=\overline{K\left(t_{2}\right)}$ for all $t_{1}$ and $t_{2}$ with $T+\nu<t_{1}<t_{2}$. This proves the theorem

The next theorem can be obtained from Theorem 1 by making use of the duality property.

Theorem 2. If $t_{1} \leq t_{2}$, then $N\left(t_{1}\right) \supseteq N\left(t_{2}\right)$. Further, $N(t)$ is independent on $t$ if $t>T+\nu$.

The proof of Theorem 1 provides a possibility to obtain an approximate null-controllability criterion for the abstract control problem.

Denote

$$
\operatorname{Im}\{\lambda I-A, B\}=\left\{\begin{array}{l|l}
z \in X \mid \begin{array}{c}
\text { there exist } x \in X \text { and } u \in U \\
\text { such that } z=(\lambda I-A) x+B u
\end{array}
\end{array}\right\} .
$$


Theorem 3. For equation (1) to be approximately null-controllable on $\left[0, t_{1}\right]$ it is necessary and, for $t_{1}>T+\nu$, sufficient that, for all $\lambda \in \sigma$,

$$
\overline{\operatorname{Im}\{\lambda I-A, \bar{B}\}}=X .
$$

Proof. Sufficiency. We obtained above that $g \in K\left(t_{1}\right)^{\perp}$ provided $t_{1}>T$ implies identity (12). By (13) we obtain from (12)

$$
\left(\Phi_{j}, g\right)=0 \quad(j \in \mathbb{N}) .
$$

This and property (III) imply $S^{*}\left(t_{1}\right) g=0$, therefore, $g \in \operatorname{Im} S\left(t_{1}\right)^{\perp}$. We have $K\left(t_{1}\right)^{\perp} \subseteq$ $\operatorname{Im} S\left(t_{1}\right)^{\perp}$, hence $\overline{\operatorname{Im} S\left(t_{1}\right)} \subseteq \overline{K\left(t_{1}\right)}$. The latter relation is equivalent to the approximate null-controllability of equation (1) on $\left[0, t_{1}\right]$. This proves the sufficiency of (13).

Necessity. If condition (13) does not hold, then there exists $\lambda \in \sigma$ and $g \in X^{*}, g \neq 0$ such that

$$
(\lambda x-A x, g)=0 \text {. for all } x \in D(A) \quad \text { and } \quad B^{*} g=0 .
$$

It follows from the latter identity that $(S(t) B u, g) \equiv 0$ for all $t \in[0,+\infty)$ and $u \in U$, but $S^{*}(t) g \neq 0$ for all $t \in[0,+\infty)$. Hence, $g \in K\left(t_{1}\right)^{\perp}$, but $g \notin \operatorname{Im} S\left(t_{1}\right)^{\perp}$. This proves the necessity of (13)

\section{Examples}

Let $X, Y$ be Hilbert spaces and let $A$ be an unbounded self-adjoint operator generating a compact semigroup $\{S(t)\}_{t \geq 0}$. In this case conditions (I) - (II) hold (see [1: pp. 176-182], all the eigenvalues $\lambda_{j} \in \sigma(j \in \mathbb{N})$ are real with multiplicity $\alpha_{j}=1$, there exists $w \in \mathbb{R}$ such that $\lambda_{j}<\omega \quad(j \in \mathbb{N})$ and $\lim _{j \rightarrow \infty} \lambda_{j}=-\infty$. The function $S(t) x$ is expressed as a series of eigenvectors, converging at $t>0$. Therefore property (III) holds with $T=0$. We assume also

$$
\sum_{j=1}^{\infty} \frac{1}{\left|\lambda_{j}\right|}<\infty .
$$

It follows from the results of [8] that provided (15) the sequence (6) is minimal on $[0, \nu]$ for any $\nu>0$. Hence, the following corollary is true which by a different method was already proved in [21].

Corollary 1. $\overline{K(t)}$ and $N(t)$ are independent of $t$ for $t \geq 0$.

Consider a linear hereditary system

$$
\begin{aligned}
& \dot{x}(t)=\int_{-h}^{0} d A(\tau) x(t+\tau)+B u(t) \\
& x(\xi)=\varphi(\xi) \quad(-h \leq \xi \leq 0) \\
& y(t)=\int_{-h}^{0} d C(\tau) x(t+\tau) \quad\left(0 \leq t \leq t_{1}\right)
\end{aligned}
$$


where $A(\cdot)$ and $C(\cdot)$ are $(n \times n)$ - and $(p \times n)$-matrices, respectively, $B$ is an $(n \times r)$ matrix, and $\varphi(\cdot) \in C^{n}[-h, 0]$. Equation (16) - (18) is described by abstract equation (1) - (3), for which the corresponding operator $A$ satisfies the conditions (I) - (II) [10], and condition (III) holds for a wide class of $A(\tau)$ (see [14: p. 101]).

Let

$$
\Delta(z)=\operatorname{det}\left\{z I-\int_{-h}^{0} d A(\tau) \exp z \tau\right\}
$$

Denote by $\omega$ the exponential of the function $\Delta(z)[13]$, i.e.

$$
\omega=\varlimsup_{|x| \rightarrow \infty} \frac{1}{|z|} \log |\operatorname{det} \Delta(z)|
$$

Lemma 1. The sequence (6) is minimal on $[0, \nu]$ for any $\nu>\omega$.

Proof. If

$$
A(\tau)=\sum_{j=0}^{m} A_{j} \chi_{\left[-h_{j+1},-h_{j}\right]}(\tau) \quad . \quad\left(0=h_{0}<h_{1}<\ldots<h_{m}=h,-h \leq \tau \leq 0\right)
$$

where $A_{j}$ are $(n \times n)$-matrices and $\chi_{\left[-h_{j+1},-h_{j}\right]}(\tau)$ is the characteristic function of the interval $\left[-h_{j+1},-h_{j}\right)$, then the above assertion follows from [22: Lemma 1]. We will prove the lemma for the general case.

It is known that

$$
\Delta(z)=\sum_{j=0}^{n} r_{j}(z) z^{j}
$$

where $r_{j}(z)$ is represented as a finite sum of products of numbers

$$
\int_{-h}^{0} d a_{j k}(\tau) \exp (-z \tau) \quad(j=1, \ldots ; n)
$$

with $a_{j k}(\tau)$ being the elements of the matrix $A(\tau)$. Hence the functions $r_{j}(\mathrm{i} y)$ with $\mathrm{i}$ $=\sqrt{-1}$ are bounded on $\mathbb{R}$. Let $f=f(t)$. $(t \in \mathbb{R})$ be an infinitely differentiable scalar function such that $f^{(k)}(t)=0(k \in \mathbb{N})$ for $t<0$ as well as $t>\delta$, let

$$
q(z)=\int_{0}^{\delta} f(t) \exp (-z t) d t \quad \text { and } \quad p(z)=\Delta(z) q(z)
$$

Since

$$
p(z)=\sum_{j=0}^{n} r_{j}(z) \int_{0}^{\delta} f^{j}(t) \exp (-z t) d t
$$

and the functions $r_{j}(i y)(j=1, \therefore, n)$ are bounded in $\mathbb{R}$, we have $\int_{-\infty}^{+\infty}|\dot{p}(i y)|^{2}<+\infty$ and $p(z)$ is of exponential type lesser or equal $\omega+\delta$ (see [13: pp. 35-37]). Hence the same is true for the function $p(z) /\left(z-\lambda_{1}\right)$ and, by virtue of [19: Theorem 1.1.1], the sequence (6) is minimal on $[0, \nu]$ for for all $\nu>\omega$, as claimed 
Therefore, if condition (III) holds for the hereditary system (16) - (18), then Theorems 1 and 2 are valid for this system (Theorems 1 and 2 for system (16) - (18) have been proved by A. W. Olbrot [15] and D. Salamon [17]).

The attainable set $K(t)$ is not necessarily closed. It immediately follows from Theorem 1 that the set $K(t)$, closed for all $t>T$, is independent of $t$ for $t>T$. This argument motivates our interest in conditions for the closeness of $K(t)$. Sufficient conditions for the closeness of $K(t)$ with respect to the topology of the space $W_{2}^{1}\left([-h, 0], R^{n}\right)$ are proved in [12] for the particular case of hereditary equation (16) - (17) with one delay,

$$
\dot{x}(t)=A_{0} x(t)+A_{1} x(t-h)+B u(t)
$$

( $A_{0}$ and $A_{1}$ are constant ( $\left.n \times n\right)$-matrices) and then are extended [4] to neutral systems (in general system (19) cannot be considered as a particular case of equation (1))

$$
\dot{x}(t)=A_{0} x(t)+A_{1} x(t-h)+A_{-1} \dot{x}(t-h)+B u(t)
$$

(see also $[2,10]$ ). However, it was proved in [3: Corollary 5.1] that for system (19) $K(t)$ is always independent of $t$ for $t>n h$.

This stronger property was generalized to the case of arbitrary $m$ in [20] for neutral systems with arbitrary numbers of point delays.

Theorems 1 and 2 and corresponding corollaries are applied for investigations of approximate controllability and observability (see, for instance, $[3,15,17,20,21]$ and the accompanying bibliographies). But these theorems are not sufficient for the investigation of complete (exact) null-controllability. Corollary 5.1 of [3] and the corresponding more general result of [20] are useful for investigations of exact null-controllability and exact controllability.

\section{Concluding remarks}

It is proved that the attainable set $K(t)$ is independent of $t$ for $t>T+\nu$, if properties (I) - (IV) hold. A new proof of the approximate null-controllability criterion for equation (1) is presented. Theorem 3 is a particular case of Theorem 2 of [21] proved for the general boundary control problem. It is shown that in the absence of a boundary control it is unnecessary to verify the independence of $\overline{K(t)}$ for sufficiently large $t$ in advance in order to obtain approximate null-controllability conditions. Property (III) and the minimality of the functions (6) provide the required independence of $t$ for $\overline{K(t)}$, and we can replace the requirement for the independence of $t$ for $\overline{K(t)}$ by property (IV). The problem of minimality conditions for exponentials' is a classical one (see, for instance, $[8,19]$ and accompanying bibliographies).

Properties of the attainable set $K(t)$ for equation (1) with unbounded input operator $B$ are not considered in the present article. The control theory for equation (1) with unbounded input operator $B$ was investigated in a number of papers (see $[6,16,18]$ and accompanying bibliographies), and their results are a reason of investigating the properties of the attainable set $K(t)$ and the unobservable set $N(t)$ for abstract control systems with unbounded input-output operators. 


\section{References}

[1] Balakrishnan, A. V.: Applied Furictional Analysis. Berlin - Heidelberg - New York: Springer-Verlag 1976.

[2] Banks, H. T. and M. O. Jacobs: An attainable set approach to optimal control of functional differential equations with function space terminal conditions. J. Diff. Eqns 13 (1973), $127-149$.

[3] Banks, H., Jacobs, M. and C. Langenhop: Characterization of the controlled states in $W_{2}^{1}$ of linear retarded systems. SIAM. J. Contr. 13 (1975), $611-649$.

[4] Bartosiewicz, Z.: Closeness on attainable set of linear neutral control systems. Control and Cybern. 8 (1979), 179 - 189.

[5] Bellman, R. and K. Cooke: Differential-Difference Equations. New York: Academic Press 1963.

[6] Curtain, R. F. and D. Salamon: Finite dimensional compensators for infinite dimensional systems with unbounded input operators. SIAM J. Control Optim. 24 (1986), 797 - 816.

[7] Fattorini, H. O.: Some remarks on complete controllability. SIAM J. Control 4 (1966), $686-694$.

[8] Fattorini, H.O. and D. L. Russel: Uniform bounds on biorthogonal functions for real exponentials with an application to the control theory of parabolic equations. Quart. Appl. Math. 32 (1974), 45 - 69.

[9] Hale, J.: Theory of Functional Differential Equations. Berlin - Heidelberg - New York: Springer-Verlag 1977.

[10] Hermes, H.: On the closure and convexity of attainable sets in finite and infinite dimensions. SIAM J. Control 5 (1967), 409 - 417.

[11] Danford, N. and J. T. Schwartz: Linear Operators, Part 1: Interscience 1957.

[12] Kurcyusz, K. and A. Olbrot: On the closure in $W_{1}^{q}$ of the attainable subspaces of linear time-lag systems. J. Diff. Equat. 24 (1977), 29 - 50.

[13] Levin, B. : Distribution of Zeroes of Entire Functions. Providence (R.I.): Amer. Math. Soc. 1964.

[14] Myshkis, A.: Linear Differential Equations with Delay Arguments (in Russian). Moscow: Nauka 1972.

[15] Olbrot, A. W.: Observability and observers for a class of linear systems with delays. IEEE Trans. Automat. Contr. AC-26 (1981), 513 - 517.

[16] Pritchard, A. I. and D. Salamon: The linear quadratic control problem for infinite dimensional systems with unbounded input and output operators. SIAM J. Contr. Opt. 25 (1987), $121-144$.

[17] Salamon, D.: On controllability and observability of time-delay systems. IEEE Trans. Automat. Contr. AC-29 (1984), 432 - 439.

[18] Salamon, D.: Infinite dimensional linear systems with unbounded control and observation: a functional analytic approach. Trans. Amer. Math. Soc. 300 (1987), 383 - 431.

[19] Sedletskii, A.: Biorthogonal expansions of functions in the series of exponents on the real interval. Russ. Math. Surveys 37 (1982), $51-95$.

[20] Shklyar, B.: On the controllability theory for neutral systems (in Russian). Izvestia Akad. Nauk BSSR, Seria Fiz.-Mat. Nauk (1980)6, 117 - 118.

[21] Shklyar, B.: Controllability of linear systems with distributed parameters. Diff. Equ. 26 (1991), 326 - 335. 
[22] Shklyar, B.: Observability of objects of neutral type by means of linear operations. Diff. Equ. 24 (1988), 196 - 201.

Received 12.07.1994 